\title{
The Complete Chloroplast Genome of a Variety of Lagenaria siceraria var. microcarpa (Naud.) Hara
}

Zhaohui Zhang ${ }^{1,2}{ }^{*}$, Dandan $\mathrm{Xi}^{2}{ }^{*}$, Jie Zhao ${ }^{3}$, Xiaofeng $\mathrm{Li}^{2}$, Lu Gao ${ }^{2}$, Chunhong Chen ${ }^{1}$, Yuying Zhu ${ }^{2}$, Hongfang Zhu ${ }^{2} \square$

1 Zhuanghang Comprehensive Experiment Station, Shanghai Academy of Agricultural Sciences, Shanghai, China

2 Horticultural Research Institute, Shanghai Academy of Agricultural Sciences, Shanghai, China

3 Shanghai Pudong New District Agro-Technology Extension Center, Shanghai, China

* These authors contributed equally to this work

$\nabla$ corresponding author email: zhuhongfang@saas.sh.cn

Molecular Plant Breeding, 2021, Vol.12, No.22 doi: $10.5376 / \mathrm{mpb} .2021 .12 .0022$

Received: 29 Apr., 2021

Accepted: 08 Jun., 2021

Published: 16 Jul., 2021

Copyright (C) 2021 Zhang et al., This is an open access article published under the terms of the Creative Commons Attribution License, which permits unrestricted use, distribution, and reproduction in any medium, provided the original work is properly cited.

Preferred citation for this article:

Zhang Z.H., Xi D.D., Zhao J., Li X.F., Gao L., Chen C.H., Zhu Y.Y., and Zhu H.F., 2021, The complete chloroplast genome of a variety of Lagenaria siceraria var. microcarpa (Naud.) Hara, Molecular Plant Breeding, 12(22): 1-4 (doi: 10.5376/mpb.2021.12.0022)

\begin{abstract}
Small gourd (Lagenaria siceraria var. microcarpa (Naud.) Hara), a cultivar of Lagenaria family, is an important medicinal and ornamental plants. However, there are few studies on its chloroplast (cp) genome. In this study, we characterized its complete chloroplast (cp) genome with high throughput sequencing. The circular cp genome is determined to be $157,121 \mathrm{bp}$ in length, with a long sing-copy and a small sing-copy separated by a pair of inverted repeat regions. A total of 132 genes were annotated, including 86 protein-coding genes, 37 tRNA genes, 8 rRNA genes, and 1 pseudo gene. The overall GC content of $\mathrm{cp}$ genome was $37.09 \%$. The maximum likelihood tree suggested that small gourd was closely related to Lagenaria siceraria. These results provided an understanding of small gourd cp genome and might be useful for future genetic studies.
\end{abstract}

Keywords Lagenaria siceraria var. microcarpa (Naud.) Hara; Chloroplast genome; Phylogenetic analysis

\section{Background}

The genus Lagenaria, well known as a member of Cucurbitaceae family, comprises Lagenaria siceraria [Mol.] Stand (L. siceraria), L. siceraria var. hispida, L. siceraria var. microcarpa, and L. siceraria var. depressa. (Ahmed and Ashiq, 2018). Numerous pharmacological activities like anticancer, antidiabetic, antihelmintics, antimicrobial, and antioxidant have been found in the extract from Lagenaria (Mohan et al., 2012; Attar and Ghane, 2018). These characteristics indicate Lagenaria plants have great medicinal values in addition to edible and ornamental values. Small gourd (L.siceraria var. microcarpa (Naud.) Hara), a variety of Lagenaria, originates in China. Except Aiying Li isolated a hypoglycemic component in small gourd, there are few researches have been made on small gourd ( $\mathrm{Li}, 2009)$. However, the genetic information like nuclear genome and chloroplast (cp) genome still remains unknown. Since plant $\mathrm{cp}$ plays essential roles in photosynthesis and various metabolic pathways, therefore, we firstly characterized the complete cp genome of small gourd and deposited the genome in GenBank (GenBank accession number: MT773628.1).

\section{Results}

We planted a cultivar of small gourd, in Zhuanghang Comprehensive Experiment Station, Shanghai, China $\left(121.391429^{\circ} \mathrm{E}, 30.894732^{\circ} \mathrm{N}\right)$ and the germplasm resource (voucher specimen: LS-133) was deposited in Shanghai Academy of Agricultural Sciences. Fresh plant leaves were obtained to extract DNA. Then, the extracted DNA was sequenced by Nanjing Genepioneer Biotechnologies Inc. (Nanjing, China) on Illumina Novaseq platform. After removing low-quality reads, obtained clean reads were used to assemble the cp genome by SPAdes version3.10.1(Bankevich et al., 2012), and gaps were filled by Gapfiller version 2.1.1 (Boetzer and Pirovano, 2012). Blast version 2.6 (https://blast.ncbi.nlm.nih.gov/Blast.cgi) was used to annotate the assembled cp genome sequence, HMMER version $3.1 \mathrm{~b} 2$ (http://www.hmmer.org/) was performed to predict rRNA, and ARAGORN version 1.2.38 (http://130.235.244.92/ARAGORN/) was used to identify tRNA. Finally, the cp genome map was generated by OGDRAW(https://chlorobox.mpimp-golm.mpg.de/OGDraw.html). 
Consequently, a total of $157,121 \mathrm{bp}$ in size of the circular cp genome of small gourd is obtained, consisting of a large single-copy (LSC) region of 86,891 bp and a simple single-copy (SSC) region of 18,012 bp (Figure 1). The LSC and SSC are separated by two invert repeat regions (IRa and IRb) of 26,146 in each region. The overall GC content is $37.09 \%$ higher than that of IR regions (16.64\%) but lower than that of LSC $(55.30 \%)$. A total of 132 genes, consisting of 86 protein-coding genes, 37 tRNA genes, 8 rRNA genes, and 1 pseudo gene, are predicted. Most of the genes occur in a single copy while7 protein-coding genes ( $n d h B, r p l 2, r p l 23, r p s 12, r p s 7, r p o C, y c f 15$, and $y c f 2), 4$ rRNA genes (rrn16, rrn23, rrn4.5, and rrn5), and 7 tRNA genes (trnA-UGC, trnl-CAU, trn-GAU, $\operatorname{trn} L-C A A, \operatorname{trnN}-G U U, \operatorname{trnR}-A C G$, and $\operatorname{trn} V-G A C)$ are duplicated. Among protein-coding genes, 8 genes (ndhA, $n d h B$, petB, petD, atpF, rpl2, rps 16, and rpoC1) contain one intron each and rps12 possess two intros.

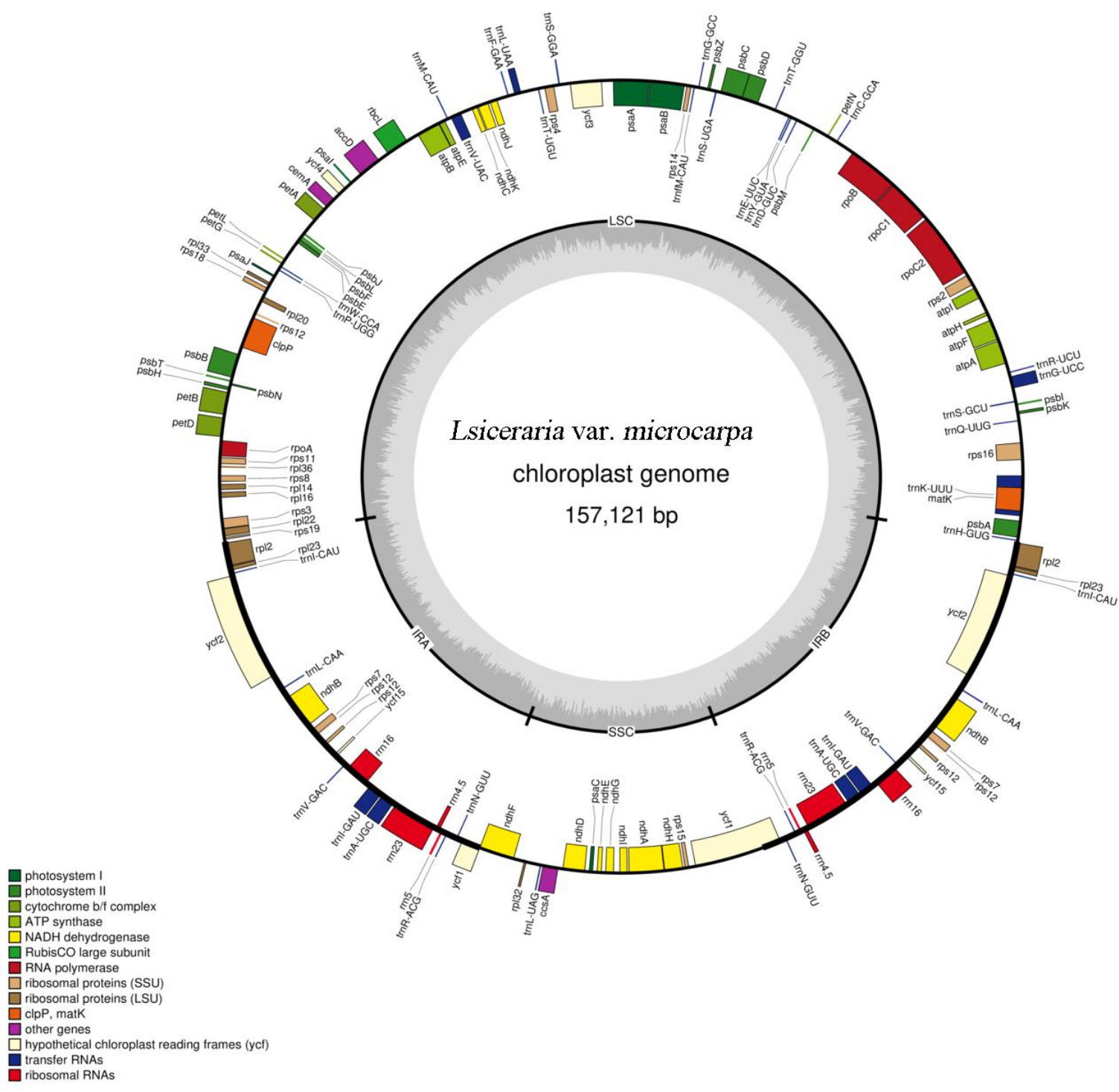

Figure 1 The cp genome map of L.siceraria var. microcarpa (Naud.) Hara.

Note: Boxes on the inside of the outermost circle represent genes clockwise transcription while boxes outside represent genes counterclockwise transcription. Dashed area in the inner circle represents GC content

To discover the phylogenic relationships of small gourd in Cucurbitaceae family, a phylogenetic analysis was performed on $11 \mathrm{cp}$ genome sequencings. A multiple sequence alignment was finished by MAFFT version 7.427 (https://mafft.cbrc.jp/alignment/software/) (Katoh and Standley, 2013), and phylogenetic tree was deduced using RAxML version8.2.10 (https://cme.h-its.org/exelixis/software.html) based on the maximum likelihood (ML) with 1000 bootstrap replicates. The ML tree reveals that small gourd is most related to $L$. siceraria with 0 variation 
coefficient and $100 \%$ bootstrap support value (Figure 2). Additionally, there is a samll variation coefficient between small gourd and Citullus lanatus (Figure 2). Our discovery will provide values for future phylogenetic studies within Lagenaria genus.

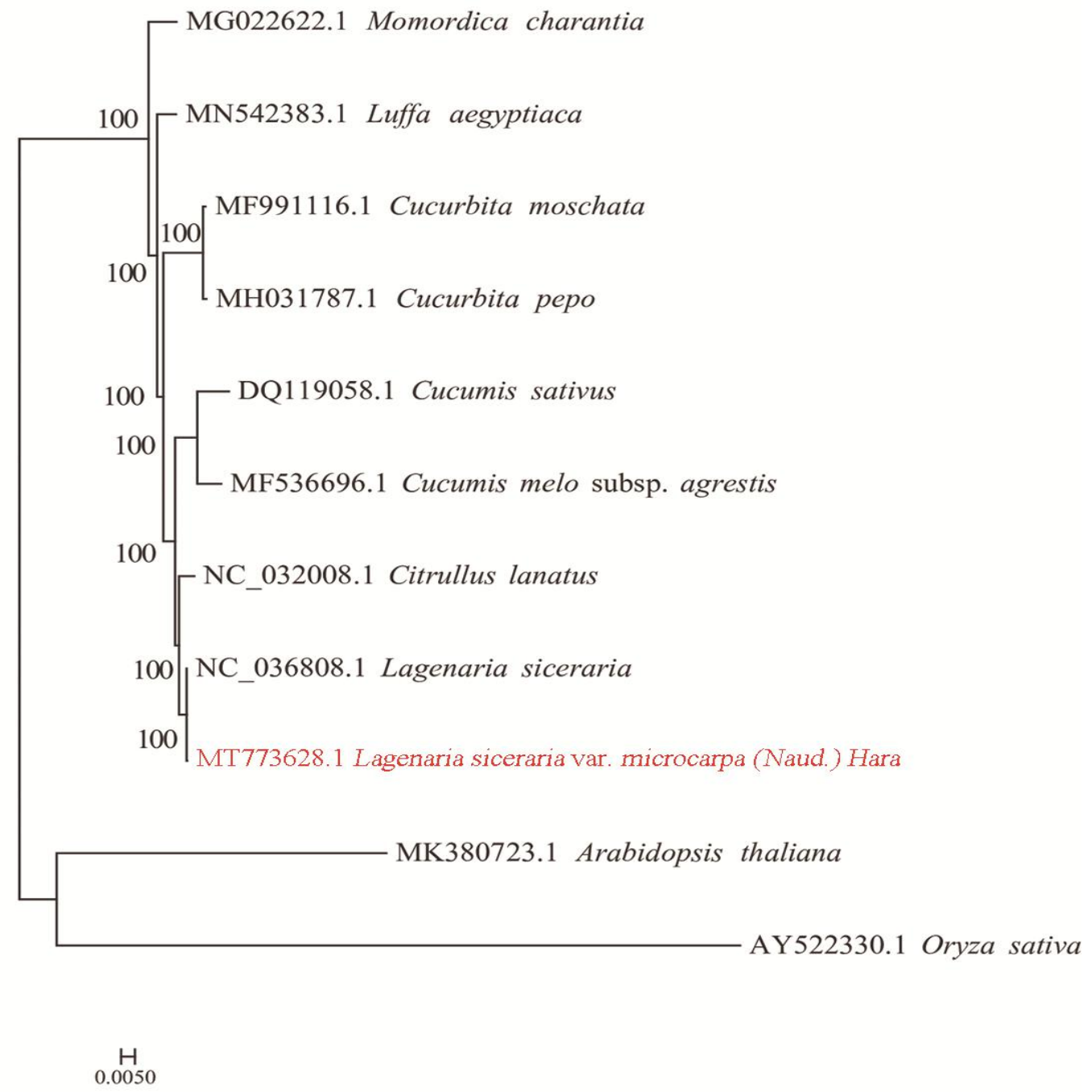

Figure 2 The phylogenetic tree of small gourd and 10 other species using whole cp genomes

Note: The bootstrap support values and GenBank accession numbers were shown at the branches

\section{Availability of Data and Material}

The genome sequence data that support the findings of this study are openly available in GenBank of NCBI at (https://www.ncbi.nlm.nih.gov/nuccore/MT773628.1/) under the accession No. MT773628.1. The associated BioProject, SRA, and Bio-Sample numbers are PRJN668886, SRR12824716, and SAMN16427048, respectively.

\section{Authors' contributions}

Zhaohui Zhang planted the small gourd for cp genome sequencing. Dandan Xi wrote this manuscript. Jie Zhao, Xiaofeng Li, Lu Gao, Chunhong Chen, and Yuying Zhu helped to prepare samples and perform the statistical analysis. Hongfang Zhu designed the study and helped to write the manuscript. All authors read and approved the final manuscript.

\section{Acknowledgments}

This study was supported by Shanghai Agriculture Applied Technology Development Program, China (Grant No. Z2017030102) and The Youth Talent Development Plan of Shanghai Municipal Agricultural System, China (Grant No. 20160105).

\section{Conflicts of interest}

No potential conflict of interest was reported by the authors 
Molecular Plant Breeding 2021, Vol.12, No.22, 1-4

http://genbreedpublisher.com/index.php/mpb

\section{References}

Ahmed D., and Ashiq N., 2018, In vitro analysis of anti-diabetic and anti-oxidative potential of pedicles of fruit-vegetable bottle gourd, Pak J Pharm Sci, 31(6): 2497-2501

Attar U.A., and Ghane S.G., 2018, Optimized extraction of anti-cancer compound-cucurbitacin I and LC-MS identification of major metabolites from wild bottle gourd (Lagenaria siceraria (Molina) Standl.), South African Journal of Botany, 119: 181-187 https://doi.org/10.1016/j.sajb.2018.09.006

Bankevich A., Nurk S., Antipov D., Gurevich A.A., Dvorkin M., Kulikov A.S., Lesin V.M., Nikolenko S.I., Pham S., Prjibelski A.D., Pyshkin A.V., Sirotkin A.V., Yahhi N.V., Tesler G., Alekseyev M.A., and Pevzner P.A., 2012, Spades: a new genome assembly algorithm and its applications to single-cell sequencing. J Comput Biol 19(5): 455-477

https://doi.org/10.1089/cmb.2012.0021

PMid:22506599 PMCid:PMC3342519

Boetzer M., and Pirovano W., 2012, Toward almost closed genomes with gapfiller. Genome Biol 13(6): 1-9 https://doi.org/10.1186/gb-2012-13-6-r56 PMid:22731987 PMCid:PMC3446322

Katoh K., and Standley D.M., 2013, Mafft multiple sequence alignment software version 7: improvements in performance and usability, Mol Biol Evol 30(4): $772-780$

https://doi.org/10.1093/molbev/mst010 PMid:23329690 PMCid:PMC3603318

Li A.Y. (2009) Study of isolation and purification of effective hypoglycemic component in bitter hyacinth (in Chinese). China Pharmaceuticals 18: 11-12

Mohan R, Birari R, Karmase A, Jagtap S, Bhutani KK (2012) Antioxidant activity of a new phenolic glycoside from Lagenaria siceraria stand. Fruits. Food Chem 132(1): 244-251

https://doi.org/10.1016/j.foodchem.2011.10.063

PMid:26434287 